\title{
ANTHROPOMETRY OF THE YOUNG ADULT COLLEGE RUGBY PLAYER IN WALES
}

\author{
W. BELL, Dip.P.E. \\ Department of Physical Education, College of Education, Cyncoed, Cardiff, Wales
}

\begin{abstract}
Twenty-one anthropometric measurements were taken on 33 first class and 29 second class young adult college rugby players. Each player was classified according to position.

There were significant differences between first and second class players in a number of measurements and it was felt necessary therefore to consider the two groups apart. Forwards were considered separately from backs.

Physique was determined according to Parnell. Overall, forwards had about a one-half unit more Fat than backs. Mean ratings for the first class forward were 4.5/5.0/2.5 and backs 4.0/5.0/2.5; second class forwards 4.5/4.5/2.5 and backs 4.0/4.5/3.0.

Lean body weight and total body fat were derived according to Wilmore and Behnke. In both groups forwards had higher lean body weights and total body fat values than backs. In the first class player percent body fat was approximately the same for forwards and backs (16\%). There was a $5 \%$ differential between second class forwards $(17 \%)$ and backs $(12 \%)$.

The sum of cross-sectional diameters of arm, calf and thigh, and of four transformed skinfolds gave similar results, for forwards and backs in both groups, as lean body weight and total body fat.
\end{abstract}

An analysis of variance was carried out within groups and between positions for all measurements. There were a greater number of significant differences within the first class player. Forwards differed, for example, in height, weight, lean body weight, total body fat and femur; backs in height, lean body weight, bi-iliac and arm length. The second class $\stackrel{0}{A}$ player differed in other measurements, for instance, humerus and upper-arm circumference. 


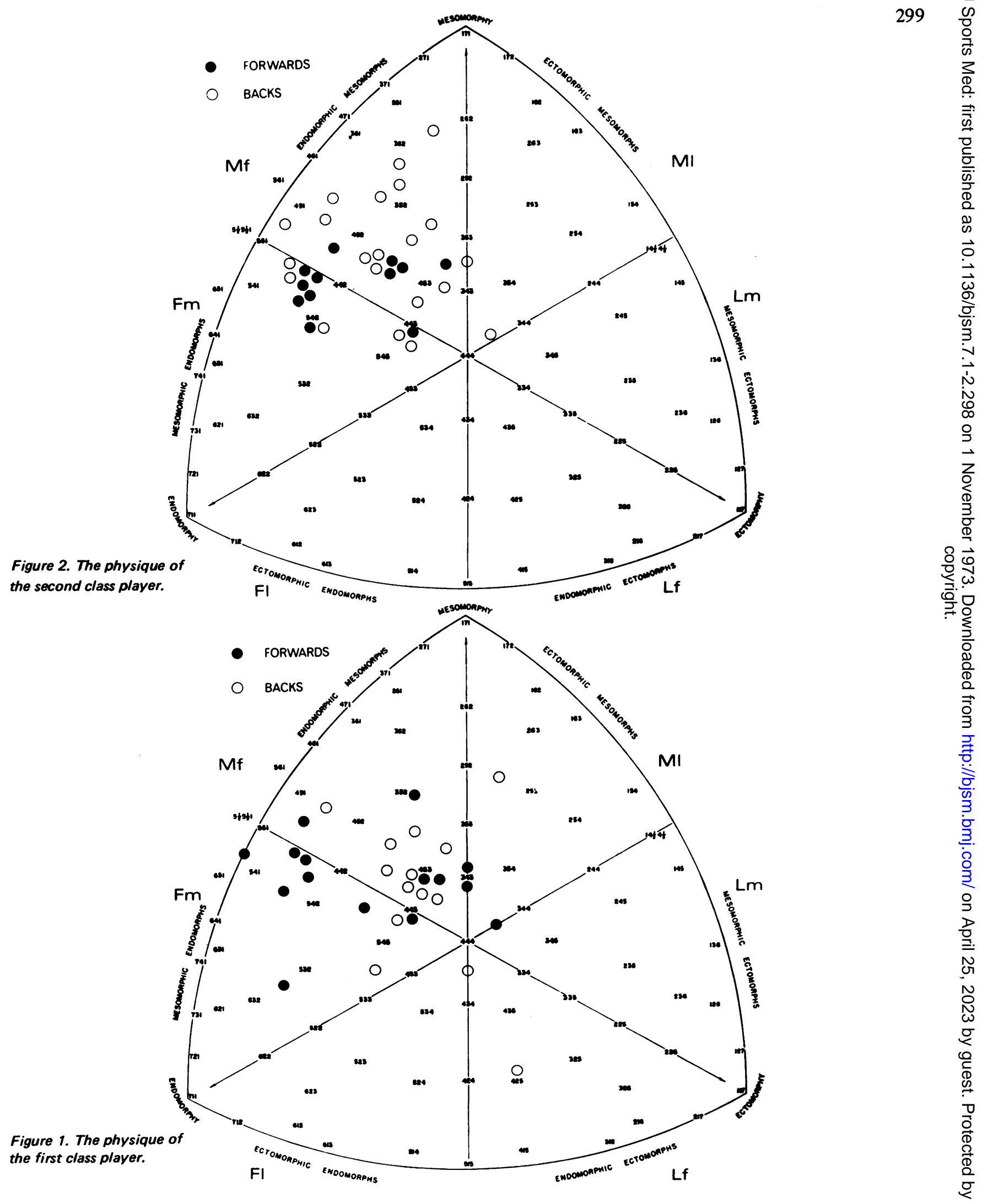

